\title{
Metabolic system dynamics: lumped and distributed models
}

\author{
G.M. Saidel ${ }^{1,2}$, J.A. DiBella II $^{1}$ \& M.E. Cabrera ${ }^{1,2,3}$ \\ ${ }^{\prime}$ Department of Biomedical Engineering, Case Western Reserve University \\ Cleveland, OH, USA \\ ${ }^{2}$ Center for Modeling Integrated Metabolic Systems \\ Case Western Reserve University \\ ${ }^{3}$ Department of Pediatrics, Rainbow Babies and Children's Hospital \\ University Hospitals of Cleveland
}

\begin{abstract}
A quantitative understanding of the complexity of cellular metabolism integrated within a tissue-organ system requires mathematical modeling and simulation. Physiologically based dynamic models that incorporate cellular metabolic reactions and transport processes of many chemical species may be spatially lumped and/or distributed. Such models allow quantitative evaluation of metabolic pathways and regulatory mechanisms under normal and abnormal conditions. In our Center for Modeling Integrated Metabolic Systems (MIMS), we are developing a general integrative whole-body model that relates cellular intermediate metabolism with responses of tissue-organ systems. These systems may be significantly affected by the spatial distribution of blood perfusion and capillary-tissue transport. As an example, we consider possible effects of spatial distribution of perfusion in skeletal muscle. In this model, blood perfusion occurs along one spatial coordinate while cellular metabolic processes are spatially lumped. In the dimensionless perfusion model, two key dimensionless parameter ratios characterize transport: axial dispersion to perfusion and capillary-tissue transport to perfusion. These have a significant effect on the behavior of the system output and the quantitative evaluation of the metabolic processes. The simulated outputs of the 1-D perfusion model are compared with those of a compartment model in which tissue is perfused isotropically.
\end{abstract}




\section{Introduction}

Essential to biological function is the coordinated and highly regulated metabolic processes occurring in all components of the human body. The human body extracts hydrocarbons from ingested food and transforms the potential chemical energy in these nutrients to ATP, which ultimately fuels all physiological processes. The regulation of energy metabolism is highly complex, even in unicellular organisms. This level of complexity at the cellular level is compounded in multi-cellular organisms. In addition to the main cellular pathways of energy metabolism, tissues and organs of a human have specialized functions with characteristic fuel requirements and metabolic patterns. To appreciate the significance of individual biochemical pathways and their regulation, these pathways must be viewed in the context of a specific tissue/organ and/or the whole organism.

A quantitative analysis of metabolic regulation, however, cannot be obtained just from in vivo experimental studies even if all necessary measurements were possible. The complexity of interacting biochemical reactions in cellular metabolism and their integration to tissue/organ and whole organism levels requires a formal theoretical framework for quantitative understanding. Such a framework must provide integration and coordination of cellular metabolism with tissue, organ, and whole-body processes. This can be achieved using sophisticated mathematical models and computer simulations. Furthermore, this framework can be applied iteratively to yield optimally designed experimental studies.

Physiologically based mathematical models incorporate cellular metabolic reactions and transport processes of numerous chemical species. In general, these dynamic models may be spatially lumped and/or distributed systems to describe highly nonlinear phenomena. Such models allow quantitative evaluation of metabolic pathways and regulatory mechanisms under normal and abnormal conditions. Consequently, such models can provide a basis for simulating the integrated effects of altering enzyme contents/activities or substrate concentrations with pharmacological agents.

The regulation of substrate and energy metabolism in human tissue/organ systems and in the whole-body is complex. Many cellular compartments, chemical species, enzymes, biochemical reactions, metabolic pathways, and control mechanisms interact with each other simultaneously to maintain ATP homeostasis and tissue/organ viability and function under stress. Metabolism of a tissue depends on its blood flow, arterial substrate and hormone levels, as well as on the metabolic, redox, energy, and activation states of its constituent cells.

Most modeling analyses of metabolism do not deal with regulation of metabolic pathways on multiple levels of organization. Complex multi-organ systems not only involve interacting metabolic pathways, but also the coordinated and integrated responses of chemical species in cells and tissues/organs of the whole body. Typically, in vivo animal experiments cannot provide direct measurements of most metabolic fluxes, which make the analysis of metabolism particularly challenging. Therefore, the development of quantitative approaches 
to the analysis of complex, multi-level metabolic systems constitutes a great challenge. Such quantitative approaches to describe, analyze, and predict the behavior of complex metabolic systems require the integration of molecular, biochemical, cellular, and physiological (organ, tissue, whole-body) data.

The regulation of whole body variables (e.g., blood glucose concentration) when a stimulus perturbs the steady state requires the response of local control mechanisms, as well as the coordination and integration of the responses of tissues/organs with different metabolic characteristics. The plasma concentrations of oxygen, metabolites (e.g., glucose), and hormones act as regulators. The net result should lead to an appropriate rate of oxygen delivery and an optimal allocation of fuels and precursors to each organ. Thus, to understand human metabolism and its regulation, it is not sufficient to have a complete knowledge of the structure and dynamics of the individual enzymes and chemical species that participate in this complex multi-level metabolic network.

An integrated multi-level systems approach is needed to quantitatively understand the dynamics of cellular metabolic processes in the context of tissue/organ systems and the whole body (Cabrera et al. [1]; Connett et al. [2]). Such an approach requires information about general features of molecular structure, enzyme kinetics, cellular metabolic control and pathways, and tissue/organ specific metabolism. This information is integrated by means of computational models, which emulate system properties that incorporate key interactions at the cellular and tissue/organ level (Fell [3]; Brand [4]; Salem et al. [5]).

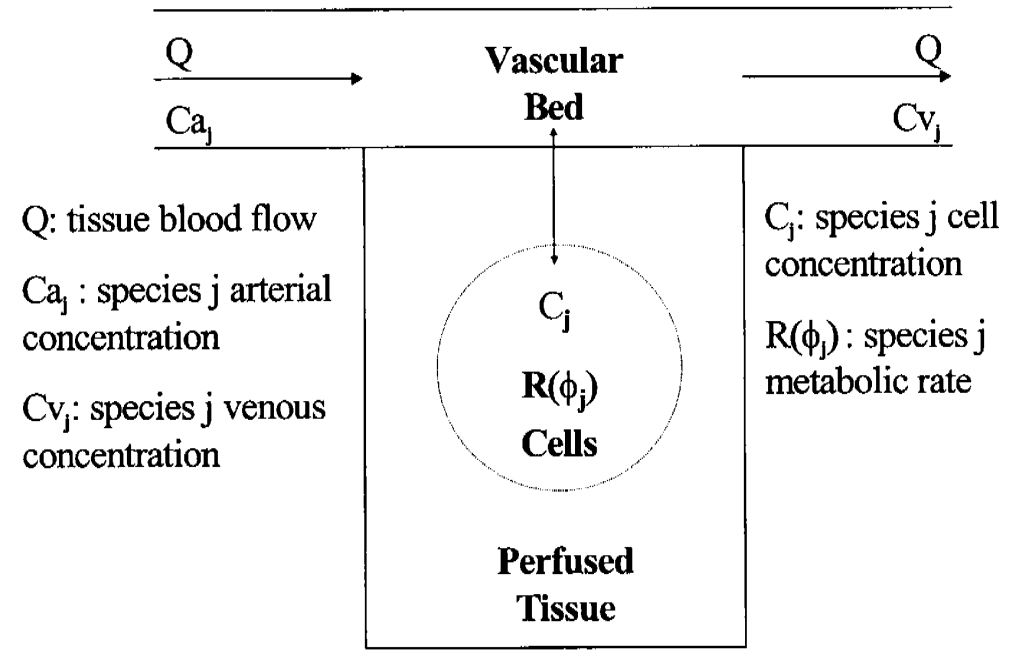

Figure 1: Key structural components of model for transport and metabolism in a blood-tissue system. 
To quantify the changes in energy metabolism and its regulation during exercise, a mathematical model of muscle bioenergetics has been developed which links various cellular metabolic processes to tissue-organ responses (Cabrera et al. [6] $\&$ [7]). In these studies, tissue has been represented by a well mixed compartment in which perfusion is assumed to be isotropic. In this work, we consider an alternative model consisting of a spatially lumped tissue perfused by blood flow in a vascular bed with one spatially continuous coordinate (Jacquez [8]). By comparing output responses produced by these models, we can determine how the effects of a spatially distributed perfusion can alter the quantitative interpretation of experimental data.

\section{Model Development}

Let us consider a metabolic model of skeletal muscle represented either as a wellmixed tissue-blood compartment or as a well-mixed tissue space in which perfusion occurs primarily along one spatial coordinate. A general schematic diagram for the structure of these models is shown in Figure. 1.

\subsection{Functional model components}

Each model is based on dynamic mass balances (compartmental and/or distributed) and mechanistic kinetics for many substrates (e.g., glucose, fatty acids) and control metabolites (e.g., ATP, NADH) through coupled reaction processes. A complete listing is given in Table 1.

Table 1: Chemical species and functional roles in metabolic model.

\begin{tabular}{|c|c|c|c|c|}
\hline Glycolysis & Krebs Cycle & $\begin{array}{l}\text { Blood-Tissue } \\
\text { Exchange }\end{array}$ & Lipolysis & $\begin{array}{l}\text { Energy Transfer } \\
\text { Processes }\end{array}$ \\
\hline $\begin{array}{l}\text { Glycogen } \\
\text { G6P } \\
\text { GAP } \\
1,3 \text { BPG }\end{array}$ & $\begin{array}{l}\text { Acetyl-CoA } \\
\text { Citrate } \\
\alpha \text {-Ketoglutarate } \\
\text { Succinyl-CoA } \\
\text { Succinate } \\
\text { Malate } \\
\text { Oxaloacetate }\end{array}$ & $\begin{array}{l}\text { Glucose } \\
\text { Pyruvate } \\
\text { Lactate } \\
\text { Palmitate } \\
\text { Glycerol } \\
\text { Alanine } \\
\text { Oxygen } \\
\text { Carbon Dioxide }\end{array}$ & $\begin{array}{l}\text { Free Species } \\
\mathrm{Pi} \\
\mathrm{H}^{+} \\
\text {Free } \mathrm{CoA}\end{array}$ & $\begin{array}{l}\text { NAD } \\
\text { NADH } \\
\text { ATP } \\
\text { ADP } \\
\text { Phosphocreatine } \\
\text { Creatine }\end{array}$ \\
\hline
\end{tabular}

Each pathway contains a number of reactions that are catalyzed by specific enzymes. In our top-down modeling approach, a set of chemical reactions contained in a particular pathway may be represented by a smaller representative set of reactions that incorporate substrates with coupled control metabolites. The reaction processes describing cellular metabolism will be modeled as stoichiometrically coupled reactions obeying the law of mass action. 


\subsection{Dynamic mass balances of chemical species.}

In a compartment model of a spatially lumped, perfused tissue, the concentration dynamics $C_{j}(t)$ of chemical species $j$ has the general form:

$$
V_{e f f} \frac{d C_{j}}{d t}=R\left(\phi_{j}\right)+Q\left[C_{a j}-\sigma_{j} C_{j}\right]
$$

where $V_{\text {eff }}$ is the effective tissue volume, $R\left(\phi_{j}\right)$ metabolic rate depending on reaction flux $\phi, Q$ tissue blood flow, $\mathrm{C}_{\mathrm{aj}}$ arterial blood concentration, and $\sigma_{\mathrm{j}}$ blood-tissue partition coefficient. Only a subset of the chemical species exists in blood as well as in tissue cells (as indicated in Table 1). For those that exist in cells only, the second term on the right side of eqn. (1) vanishes. In dimensionless form, the model yields a dimensionless parameter group that involves the characteristic transit time $\left(\mathrm{V}_{\text {eff }} / \mathrm{Q}\right)$.

For a well-mixed tissue space perfused by one-dimensional blood flow, the governing equation for species concentration $\mathrm{C}_{\mathrm{bj}}$ in the vascular bed is

$$
\frac{\partial C_{b j}}{\partial t}+Q \frac{\partial C_{b j}}{\partial v}=A^{2} D_{j} \frac{\partial^{2} C_{b j}}{\partial \nu^{2}}-\frac{P_{j} S}{V_{b}}\left[C_{b j}-\sigma_{j} C_{t j}\right]
$$

where position along the vascular volume $\mathrm{V}_{\mathrm{b}}$ is indicated by volume variable $\mathrm{v}$ $\left(0<v<V_{b}\right), A$ is vascular cross-section area, $D_{j}$ is a dispersion coefficient, $P_{j}$ is vascular permeability, and $S$ is vascular surface area for blood-cell transport. The boundary conditions for eqn. (2) assume that the input concentrations of chemical species are known and that the concentration gradients at the output are negligible.

The governing equation for species concentration $\mathrm{C}_{\mathrm{tj}}$ in the well-mixed peri-vascular tissue is

$$
V_{t c} \frac{d C_{t j}}{d t}=\frac{P S}{V_{b}} \int_{0}^{V_{b}}\left[C_{b j}-\sigma_{j} C_{t j}\right] d v+R\left(\phi_{j}\right)
$$

where $V_{t c}$ is the volume of tissue cells. In dimensionless form, this model yields dimensionless parameter groups that characterize the relative importance of axial dispersion to perfusion (or "convection"), $\mathrm{A}^{2} \mathrm{D}_{\mathrm{j}} / \mathrm{QV}$, and the relative importance of blood-tissue transport to perfusion, $\mathrm{P}_{\mathrm{j}} \mathrm{S} / \mathrm{Q}$.

If we integrate eqn. 2 over all $\mathrm{v}$ and assume equilibrium between the average blood concentration and tissue concentration of exchangeable species, then combining eqns. 2 and 3 leads to an equivalent system model:

$$
V_{j} \frac{d C_{s j}}{d t}=R\left(\phi_{j}\right)+Q\left[C_{a j}-\sigma_{j} C_{s j}\right]
$$


where the equivalent system volume is $\mathrm{V}_{\mathrm{j}}=\mathrm{V}_{\mathrm{tc}}+\sigma_{\mathrm{j}} \mathrm{V}_{\mathrm{b}}$ and the equivalent system concentration of species $j$ is $C_{s j}$. Comparison of eqn. 1 with eqn. 4 indicates that these are equivalent if $\mathrm{V}_{\text {eff }}=\mathrm{V}_{\mathrm{j}}$.

For species with molecular conservation in cells (viz., $\mathrm{NADH}, \mathrm{PCr}$, ATP), we obtain stoichiometric relationships, e.g., $\mathrm{C}_{\mathrm{NAD}}+\mathrm{C}_{\mathrm{NADH}}=$ constant.

\subsection{Metabolic reaction rates}

The metabolic reaction rate of species $\mathrm{j}$ is the net rate of production (gain) and utilization (loss):

$$
R_{j}=\sum_{k=1}^{n} \beta_{i j} \sum_{p=1}^{q} \phi_{i j}^{p}-\sum_{k=1}^{m} \beta_{j i} \sum_{p=1}^{q} \phi_{j i}^{p}
$$

where $\phi_{i j}{ }^{p}$ is the flux of species $j$ from species $i$ in reaction $p$ and $\beta_{i j}$ is the corresponding stoichiometric coefficient. For example, the reaction rate of acetylCoA is expressed as:

$$
\mathrm{R}\left(\phi_{\mathrm{AC}}\right)=\phi_{\mathrm{PYR}} \rightarrow_{\mathrm{AC}}+8 \phi_{\mathrm{FAC}} \rightarrow_{\mathrm{AC}}-\phi_{\mathrm{AC}} \rightarrow_{\mathrm{CIT}}
$$

Typically, reaction fluxes of substrates coupled with control metabolites follow Michaelis-Menten kinetics with product inhibition:

$$
\phi_{i j}=K_{i j}\left[\frac{X}{K_{X}+X}\right]\left[\frac{C_{i}}{K_{i o}}\right]\left[1+\frac{C_{i}}{K_{i o}}+\frac{C_{j}}{K_{j o}}\right]^{-1}
$$

where $\mathrm{Ks}$ are coefficients and $X$ represents a metabolic control ratio (e.g., $\mathrm{RS}=\mathrm{NADH} / \mathrm{NAD}$ ). A control ratio is included in the flux expression only if it is directly coupled to the corresponding substrate reaction. As an example, consider pyruvate reduction to lactate coupled with NADH utilization:

$$
\text { PYR } \rightarrow \text { LAC; NADH } \rightarrow \mathrm{NAD}^{+}
$$

The reaction flux of pyruvate reduction is represented as:

$$
\left.\left.\phi_{P L}=K_{P L}\left[\frac{R S}{K_{R S}+R S}\right] \frac{C_{P Y R}}{K_{P Y R}}\right] 1+\frac{C_{P Y R}}{K_{P Y R}}+\frac{C_{L A C}}{K_{L A C}}\right]^{-1}
$$

\section{Simulation Methods}

\subsection{Numerical methods}

The simpler model with a lumped, perfused tissue consists of coupled ordinary differential and algebraic equations. These equations constitute an initial-value problem with sparse-stiff systems, which are solved numerically with an appropriate integrator, LSODES (Hindmarsh, [9]). The more general model having a lumped tissue perfused by a one-dimensional vascular bed involves coupled partial and ordinary differential equations together with algebraic 
equations. For numerical solution, we discretize the spatial derivatives incorporating the boundary conditions using DSS/2 (Schiesser \&. Silebi [10]). The integral term in the model is discretized using Simpson's rule. Consequently, we end up with a sparse-stiff initial-value problem that is solved with LSODES.

\subsection{Simulation strategy}

Model simulations provide the basis for determining when the two models yield output responses that are similar and when they are different. This is accomplished by comparing input-output responses dynamically and at steady state. The most readily accessible output from experimental measurements come from concentrations of various species leaving the tissue in venous blood, $\mathrm{C}_{\mathrm{bj}}\left(\mathrm{V}_{\mathrm{b}}, \mathrm{t}\right)=\mathrm{C}_{\mathrm{vj}}$. However, to understand the distinctive processes that occur in the distributed model, we must also examine the dynamic changes of tissue concentration and of concentration distribution in the vascular bed.

We can examine the effects of inputs and parameters by changing the arterial blood (input) concentrations of key chemical species and by changing system parameters such as permeability, axial dispersion, and tissue volume. We start with a reference set of parameter values for which the simpler lumped model simulates experimental measurements. Then, we estimate the axial dispersion and permeability parameters $\left(D_{j}, P_{j}\right)$ of the more general distributed model whose venous blood output corresponds to that of the simpler model at steady state. This establishes the initial conditions used to examine the dynamic response to a change in a species concentration of the arterial blood. This process is repeated with different values for $D_{j}$ and $P_{j}$. As an example, we show the effects of a step decrease of oxygen concentration in the arterial blood for different values of the permeability.

\section{Results}

We examine simulations of the two models, that is with and without a spatially distributed vasculature, when the arterial oxygen concentration is reduced for 30 min. With the distributed vasculature, a higher permeability requires a higher oxygen concentration in the system to maintain a steady state (Figure 2). At 5 and $25 \mathrm{~min}$, the vascular concentration profiles are nearly the same for higher permeability because the oxygen concentrations in blood and tissue approach tissue-blood equilibrium quickly and not far from the arterial blood input. At the reference permeability value, the arterial-venous oxygen concentration difference at steady state is larger because the venous blood reflects the lower tissue concentration (Figure 3). In venous blood, the initial rate of oxygen decrease is greater with the higher permeability (Figure 4 ). The dynamic venous oxygen responses of the two models are essentially identical in the reference case, even though the vascular oxygen profiles are different (Figure 2). 


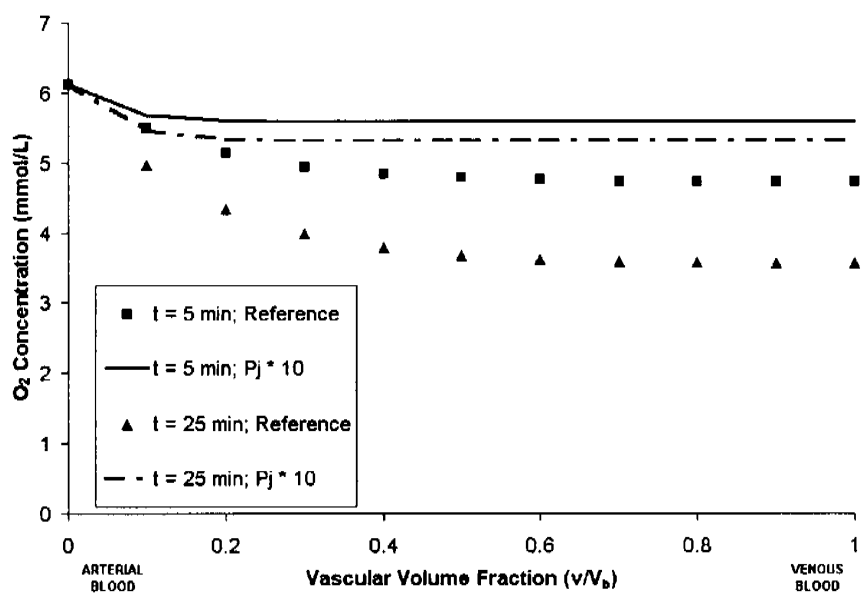

Figure 2: Effect of permeability on vascular bed oxygen concentration profile at two times after $30 \%$ step reduction in arterial oxygen concentration.

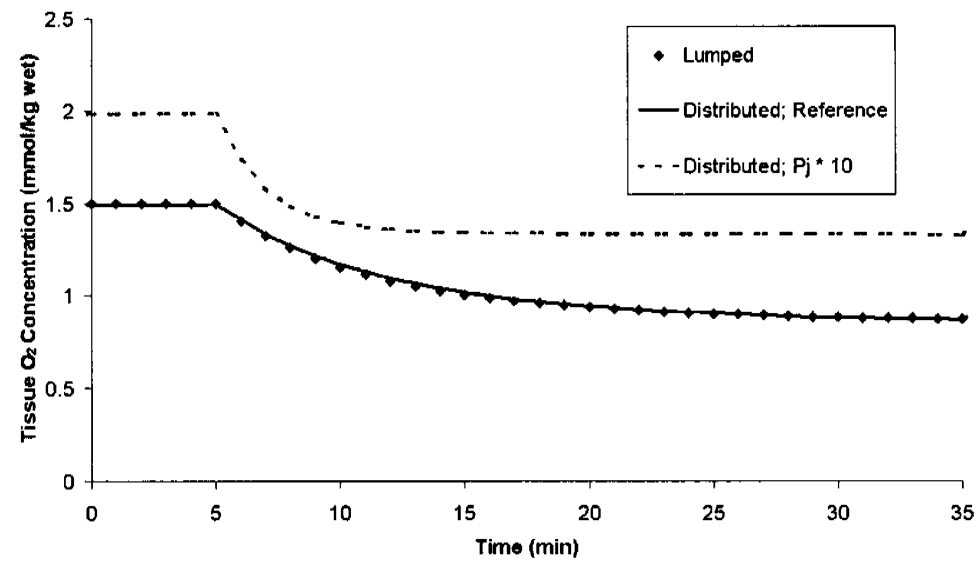

Figure 3: Effect of permeability on tissue oxygen concentration after $30 \%$ step reduction in arterial oxygen concentration. 


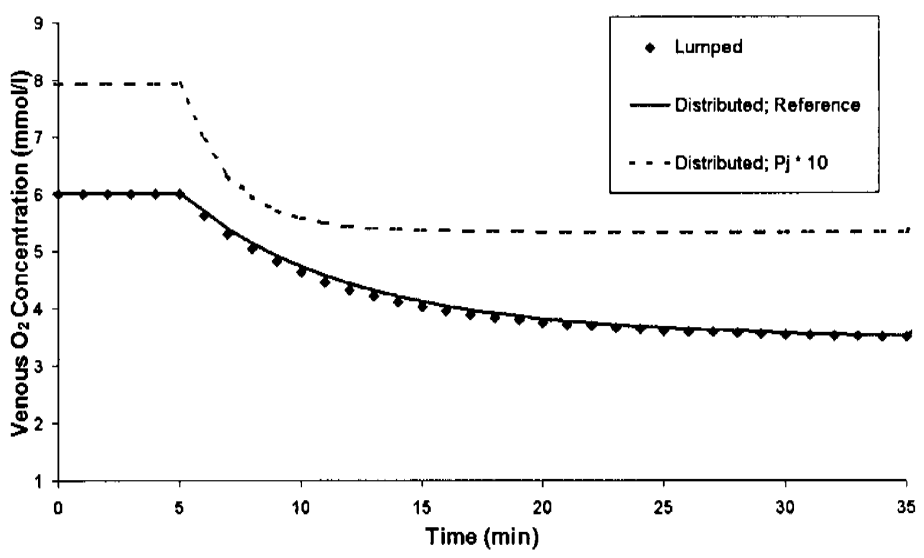

Figure 4: Effect of permeability on venous oxygen concentration after $30 \%$ step reduction in arterial oxygen concentration.

\section{Discussion}

We chose to simulate the effects of a spatially distributed vasculature on metabolic system outputs in skeletal muscle. For this tissue, a large body of published experimental studies provides key information for estimating model parameters and comparing outputs. Metabolic rate parameters that are the same for both models are estimated using data from in vitro and in vivo studies, mostly under steady-state conditions.

Transport parameters of the model with a spatially distributed vascular bed are estimated by examining terms of the dimensionless model. Parameters are chosen such that each term of the model is expected to show an effect on the output responses. Although the parameter values are unlikely to be unique, simulated model outputs are expected to correspond to experimental data within measurement error.

The lumped tissue-vascular model can simulate the venous output response well under limited conditions. Under more general conditions that can occur, for example with vascular disease, the lumped model cannot simulate the output. Furthermore, based on parameter values of the simpler model, the interpretation of the output may be physiologically inappropriate. For example, dynamic responses may require arbitrary changes of $V_{\text {eff. }}$ Comparison of the integrated distributed model with the simpler model makes evident the various assumptions associated with the latter including the basis for $V_{\text {eff. Finally, the }}$ simpler model cannot adequately simulate steady-state output changes with permeability for chemical species that have a high uptake rate in tissue and large arterial-venous (A-V) difference such as oxygen. 


\section{Acknowledgements}

This work was supported in part by grants from the National Space Biomedical Research Institute (IHF 00205) and National Institute of General Medical Sciences, NIH (GM-66309). K. Radhakrishnan provided valuable guidance for the numerical simulation.

\section{References}

[1] Cabrera, M.E., Saidel, G.M., \& Kalhan, S.C., Modeling metabolic dynamics from cellular processes to organ and whole body responses. Prog. Biophys. Molec. Biol. 69, pp. 539-557, 1998.

[2] Connett, R.J., Honig, C.R., Gayeski, T.E.J., \& Brooks, G.A., Defining hypoxia: a systems view of $\mathrm{VO}_{2}$, glycolysis, energetics, and intracellular $\mathrm{PO}_{2}$. J. Appl. Physiol. 68:833-842, 1990.

[3] Fell, D., Understanding the Control of Metabolism, Portland Press: London, 1997.

[4] Brand, M.D., Regulation analysis of energy metabolism. J. Exp. Biol. 200, pp. 193-202, 1997.

[5] Salem, J.E., Saidel, G.M., Stanley, W.C., \& Cabrera, M.E., Mechanistic model of myocardial metabolism under normal and ischemic conditions. Ann. Biomed. Eng. 30, pp. 202-216, 2002.

[6] Cabrera, M.E., Saidel, G.M., \& Kalhan, S.C., Role of $\mathrm{O}_{2}$ in regulation of lactate dynamics during hypoxia: mathematical model and analysis, $A n n$ Biomed Eng, 26, pp. 1-27, 1998.

[7] Cabrera, M.E., Saidel, G.M., \& Kalhan, S.C., Lactate metabolism during exercise: analysis by an integrative systems model. Am. J. Physiol. 277, R1522-R1536, 1999.

[8] Jacquez, J., Compartment Analysis in Biology and Medicine (2nd ed), University of Michigan Press: Ann Arbor: pg. 175, 1985.

[9] Hindmarsh, A.C., ODEPACK, A systemized collection of ode solvers. Scientific Computing. ed. R. Stepleman, North-Holland: Amsterdam, 1983.

[10] Schiesser, W.E. \& Silebi, C.A., Computational Transport Phenomena, Cambridge University Press, 1997. 